Klimaschutzpotenziale der Abfallwirtschaft

\title{
Ein großer Beitrag zum Klimaschutz ist möglich
}

\begin{abstract}
Die bisherigen Klimaschutzleistungen der Abfallwirtschaft und weitere zukünftige Potenziale in Deutschland wurden in einer Studie des Bundesverbands der Deutschen Entsorgungs-, Wasser- und Rohstoffwirtschaft e.V. (BDE), des Umweltbundesamtes und des Bundesumweltministeriums untersucht. Ein Interview mit Jochen Flasbarth
\end{abstract}

ÖkologischesWirtschaften: Herr Flasbarth, welche Bedeutung hat die Abfallwirtschaft für den Klimaschutz?

Jochen Flasbarth: Eine fortschrittliche Abfallwirtschaft leistet beachtliche Beiträge zum Klimaschutz. In Deutschland konnten die Treibhausgasemissionen durch Maßnahmen in der Abfallwirtschaft zwischen 1990 und 2006 um rund 56 Millionen Tonnen Kohlendioxidäquivalente gemindert werden. Das entspricht fast einem Viertel der in diesem Zeitraum erreichten Gesamtminderung in Deutschland.

ÖW: Findet die Abfallwirtschaft genügend Aufmerksamkeit in der öffentlichen Diskussion über Klimaschutz?

In dieser Hinsicht haben wir Nachholbedarf. Dies liegt erstens daran, dass das Thema Klimaschutz in der Öffentlichkeit mit anderen Themenfeldern wie Erneuerbare Energien und Energieeinsparmaßnahmen, zum Beispiel im Gebäudebereich, gut besetzt ist. Zweitens hat der Gesetzgeber in Deutschland für die Abfallwirtschaft die entscheidenden Weichen bereits in der Vergangenheit gestellt; damit meine ich vor allem das Ablagerungsverbot für unbehandelte Siedlungsabfälle, das im Juni 2005 in Kraft getreten ist. Dazu gehört auch der stete Ausbau des Recyclings und der energetischen Verwertung.

Die Klimaschutzpotenziale der Abfallwirtschaft in Deutschland sind bereits weitgehend genutzt; international gesehen sind allerdings noch große Potenziale vorhanden. Dies gilt für industriali- sierte Länder, die weiterhin große Mengen unbehandelter Abfälle deponieren wie zum Beispiel die USA, aber auch für Schwellen- und Entwicklungsländer, wo das Abfallaufkommen zum Teil rasant steigt. Dass durch effiziente Abfallbewirtschaftung ein großer Beitrag zum Klimaschutz geleistet werden kann, wollen wir in Zukunft in unserer internationalen Zusammenarbeit noch stärker verdeutlichen.

ÖW: In der Studie wurde die Methode der Ökobilanzierung genutzt, um die Klimaschutzpotenziale der Abfallwirtschaft zu ermitteln. Was sind die Chancen und Grenzen der Methode?

Mit der Ökobilanzierung ist es möglich, die Umweltauswirkungen von verschiedenen Abfallbehandlungsmethoden miteinander zu vergleichen. Da die Methode genormt ist, werden gut verifizierbare Ergebnisse erreicht.

Es können nahezu alle Schritte der Abfallwirtschaft, wie Sammlung, Aufbereitung und Verwertung berücksichtigt werden. Dabei werden die Belastungen durch die Abfallbehandlung und die Einsparungen durch zur Verfügung gestellte Sekundärrohstoffe oder Energie gegeneinander aufgerechnet. Im Rahmen der Ökobilanzierung werden somit insbesondere die Erfolge einer geordneten, auf das Schließen von Kreisläufen ausgerichteten Abfallwirtschaft aufgezeigt, aber auch die Belastungen einer einfachen Deponierung der Abfälle aufgedeckt.

Grenzen ergeben sich durch die Vorgabe, dass die Abfälle bei der „Abfall-Öko- bilanzierung " als gegeben angesehen und ohne Belastungen in das System eingehen. Das bedeutet: Die Erfolge der Abfallvermeidung können durch diese Methode nicht abgebildet werden.

ÖW: Das Gesamtabfallaufkommen in Deutschland beträgt 372, 9 Millionen Tonnen im Jahr. Siedlungs- und Altholzabfälle machen noch nicht einmal 15 Prozent der Gesamtmenge aus. Warum wurden in der Studie gerade diese Bereiche untersucht?

Siedlungsabfälle trugen im Abfallbereich in der Vergangenheit maßgeblich zur Klimabelastung bei; so wurden durch die Siedlungsabfallwirtschaft im Jahr 1990 noch rund 38 Millionen Tonnen Kohlendioxidäquivalente freigesetzt - insbesondere infolge hoher Methanemissionen aus Siedlungsabfalldeponien. Die Entwicklung dieses besonders klimarelevanten Bereichs der Abfallwirtschaft steht daher seit Langem im Mittelpunkt unserer Betrachtungen.

Andere Abfallarten, zum Beispiel Bauund Abbruchabfälle, die mit etwa 200 Millionen Tonnen den größten Abfallstrom am Gesamtaufkommen ausmachen, tragen nicht oder kaum zur Bildung von Treibhausgasen bei, weil hier nur wenig organisches Material, das zu Methanemissionen führen kann, vorhanden ist. Allerdings können auch hier indirekt Beiträge zum Klimaschutz durch Verwertung der in den Abfällen enthalten Rohstoffe geleistet werden. Zu diesen Rohstoffen gehören beispielsweise Metalle, Kunststoffe oder Baustoffe.

Da wir nicht nur theoretische Potenziale ermitteln, sondern auch Handlungsoptionen erarbeiten wollten, haben wir uns auf die Siedlungsabfallwirtschaft beschränkt. Es gibt aber aus dem Umweltbundesamt (UBA) und von anderen Institutionen Studien zu weiteren Abfallströmen, zum Beispiel Bau- und Abbruchabfällen, bei denen neben Klimaschutzfragen vor allem Aspekte der Ressourcenschonung im Vordergrund stehen.

Aufgrund der Komplexität der Datenerhebung der anfallenden Abfälle und deren Verbleib in Verwertung oder Behandlung befasst sich diese Studie „nur“ mit Siedlungsabfall und Altholz. 
ÖW: Die Emissionen klimaschädlicher Gase durch Siedlungsabfälle konnten zwischen 1990 und 2006 bereits erheblich gesenkt werden. Woran lag das?

In erster Linie an der Einführung abfallwirtschaftlicher Strukturen und Regelungen, die auf geschlossene Kreisläufe zielen. Dieser Ansatz wurde in Deutschland maßgeblich schon zu Beginn der 90er Jahre des letzten Jahrhunderts entwickelt. In diesem Zusammenhang sind vor allem die Einführung der Produktverantwortung, die Getrennterfassung recyclebarer Abfälle, die Vorbehandlung der Restabfälle vor deren Ablagerung sowie die geordnete Abfallablagerung mit Deponiegaserfassung und -nutzung zu nennen. Als wichtige Maßnahme möchte ich auch das seit Juni 2005 geltende Ablagerungsverbot für unbehandelte Siedlungsabfälle auf Deponien nennen.

Parallel dazu sind die Mengen an getrennt gesammelten Abfällen wie zum Beispiel Papier, Glas, Kunststoffe und Bioabfall gestiegen. Dadurch werden bei der stofflichen oder energetischen Verwertung Primärrohstoffe und Energie eingespart, was sich positiv auf die Klimabilanz auswirkt.

ÖW: Wo liegen noch ungenutzte Klimaschutzpotenziale?

Laut unserer Studie kann der Beitrag zur Treibhausgasminderung der Siedlungsabfallwirtschaft in Deutschland bis 2020 noch um weitere zehn Millionen Tonnen $\mathrm{CO}_{2}$-Äquivalente gesteigert werden. Dabei haben wir angenommen, dass 50 Prozent der noch im Restabfall vorhandenen Wertstoffe getrennt erfasst und verwertet werden. In der Detailbetrachtung werden die bedeutendsten Einsparungen durch das Recycling von Altpapier und Altholz erreicht, gefolgt vom Recycling der Leichtverpackungen und der energetischen Nutzung des Restmülls in Müllverbrennungsanlagen (MVA). Bei der MVA bietet vor allem eine gesteigerte Nutzung der anfallenden Wärme noch ein erhebliches Potenzial.

ÖW: Ist die deutsche Abfallwirtschaft ein Vorbild für andere Staaten?

Unsere Studien zum Klimaschutzpotenzial der Abfallwirtschaft stoßen besonders im Ausland auf großes Interes- se. Die Erfolge bei der getrennten Sammlung und hohe Recyclingquoten sowie effiziente Behandlungsverfahren der Restabfälle sind vorbildlich. Das Ziel geschlossener Kreisläufe wird in Zeiten der Ressourcenverknappung weltweit als besonders wichtig angesehen. Das deutsche Angebot an erprobten technischen Anlagen zur Aufbereitung und Behandlung von Abfällen ist vielfältig und kann auf die Bedürfnisse der Zielländer zugeschnitten werden. Wichtig ist jedoch nicht nur der Export von Hardware, sondern vor allem von Know-how. Im Rahmen des Techniktransfers ist das UBA besonders beim Aufbau des gesetzlichen Rahmens und der Verwaltungsstrukturen ein gefragter Partner.

ÖW: Was sind die größten Probleme in anderen Ländern?

Es gibt natürlich große Unterschiede zwischen industrialisierten Ländern und den Schwellen- und Entwicklungsländern. Allerdings ist selbst in der Europäischen Union, wo wir einem gemeinsamen gesetzlichen Rahmen unterliegen, der technische und organisatorische Stand in der Abfallwirtschaft sehr unterschiedlich und viele Länder in Europa haben noch ein großes, bisher nicht erschlossenes Klimaschutzpotenzial. Wie unsere Erfahrungen zeigen, kann man mit abfallwirtschaftlichen Maßnahmen schnell und kostengünstig Treibhausgase vermindern. Investitionen in den Aufbau einer Klima schonenden Abfallwirtschaft sind also äußerst sinnvoll.

ÖW: Welche Rolle spielen technische Innovationen für die Abfallwirtschaft der Zukunft?

Wir sind in Deutschland bereits auf einem hohen technischen Standard in der Abfallwirtschaft angekommen. Technische Innovationen in Quantensprüngen sind wohl unwahrscheinlich. Aber zum Beispiel lag bisher die Rückgewinnung von Edel- und Sondermetallen zu wenig im Fokus des Recyclings. Die zukünftige Verfügbarkeit dieser Metalle, die vor allem in Elektro- und Elektronikgeräten, zum Beispiel der Informations- und Kommunikationstechnik enthalten sind, und die Notwendigkeit, die Umweltauswirkungen des Rohstoffabbaus zu ver- ringern, stehen bei diesem Thema im Mittelpunkt unserer Betrachtungen. Die Ausrichtung des Recyclings auf die Rückgewinnung der Rohstoffe im Abfall wird noch große Anstrengungen erfordern. Wir erwarten uns davon Fortschritte bei der Schonung natürlicher Ressourcen. Eine erhöhte Ressourceneffizienz ist wesentlich für die Wettbewerbsfähigkeit unserer Wirtschaft und ein zentrales Thema für unsere Gesellschaft. Das betrifft nicht nur die Abfallwirtschaft, sondern zielt auch auf Produktions- und Konsummuster. In diesen Bereichen sind grundlegende Veränderungen notwendig. Das wird auch Auswirkungen auf die Abfallwirtschaft haben als ein Glied in der Kette geschlossener Stoffkreisläufe.

ÖW: Die Leistung der Abfallwirtschaft in allen Ehren - der beste Klimaschutz ist die Abfallvermeidung. Wie geht es damit voran?

Auf die Abfallvermeidung wird auch seitens der Europäischen Union ein verstärktes Augenmerk gelegt. Gegenwärtig erarbeiten wir die wissenschaftliche Grundlage zur Aufstellung eines Abfallvermeidungsplans. Allerdings lässt sich Abfallvermeidung nur bedingt aus der Abfallwirtschaft heraus steuern. Hier muss vor allem in den vorgelagerten Schritten von Produktion und Konsum noch viel Arbeit geleistet werden. Bisher wurden die Erfolge einer effizienteren Herstellung meistens durch verstärkten Konsum überkompensiert. Um eine Senkung der Kohlendioxidemissionen um 80 Prozent bis $2050 \mathrm{zu}$ erreichen, müssen wir unsere Wirtschafts- und Lebensweise grundlegend ändern. Der Aufbau einer „Green Economy“ steht daher ganz oben auf der Tagesordnung.

I AUTOR + KONTAKT

Jochen Flasbarth ist Präsident des Umweltbundesamtes.

Umweltbundesamt, Wörlitzer Platz 1, 06844 Dessau-Roßlau. Tel.: +49 340 2103-0, E-Mail: info@umweltbundesamt.de, Internet: www.umweltbundesamt.de 


\section{Lizenzhinweis}

Die Beiträge in ÖkologischesWirtschaften werden unter der Creative-Commons-Lizenz "CC 4.0 Attribution Non-Commercial No Derivatives" veröffentlicht. Im Rahmen dieser Lizenz muss der Autor/Urheber stets genannt werden, das Werk darf nicht bearbeitet, abgewandelt oder in anderer Weise verändert und außerdem nicht kommerziell genutzt werden. Die digitale Version des Artikels bleibt für zwei Jahre Abonnent/innen vorbehalten und ist danach im Open Access verfügbar. 